\title{
BMJ Open Prevalence of insufficient weight loss 5 years after Roux-en-Y gastric bypass: metabolic consequences and prediction estimates: a prospective registry study
}

To cite: Brissman $M$, Beamish AJ, Olbers T, et al. Prevalence of insufficient weight loss 5 years after Roux-en-Y gastric bypass: metabolic consequences and prediction estimates: a prospective registry study. BMJ Open 2021;11:e046407. doi:10.1136/ bmjopen-2020-046407

- Prepublication history and supplemental material for this paper is available online. To view these files, please visit the journal online (http://dx.doi org/10.1136/bmjopen-2020046407).

Received 28 0ctober 2020 Revised 01 February 2021 Accepted 17 February 2021

Check for updates

(C) Author(s) (or their employer(s)) 2021. Re-use permitted under CC BY. Published by BMJ.

For numbered affiliations see end of article.

Correspondence to Dr Markus Brissman; markus.brissman@ki.se

\section{ABSTRACT}

Objective The study aimed to investigate the heterogeneity of weight loss 5 years after Rouxen-Y gastric bypass (RYGB) and the association with cardiometabolic health as well as to model prediction estimates of surgical treatment failure.

Design Retrospective analysis of prospectively collected data from the Scandinavian Obesity Surgery Registry (SOReg).

Setting 29 surgical units from the whole of Sweden contributed data. Inclusion was restricted to surgical units with a retention rate of $>60 \%$ five years postsurgery.

Participants 10633 patients were extracted from SOReg. In total 5936 participants were included in the final sample, $79.1 \%$ females. The mean age of participants before surgery was $39.4 \pm 9$ years and mean body mass index (BMI) 42.9 \pm 5.1 . 2322 were excluded (death before the 5-year follow-up $(n=148)$, other types of surgery or reoperations ( $n=637$ ), age at surgery $<18$ or $>55$ years $(\mathrm{n}=1329)$, presurgery $\left.\mathrm{BMl}<35 \mathrm{~kg} / \mathrm{m}^{2}(\mathrm{n}=208)\right)$. In total, $2375(29 \%)$ of eligible individuals were lost to the 5 -year follow-up.

Main outcome The occurrence of surgical treatment failure 5 years after surgery was based on the three previously published definitions: per cent excess BMI loss $<50 \%$, total weight loss $<20 \%$ or $\mathrm{BMI}>35$ where initial BMI was $<50$, or $>40$ where initial BMI was $>50$. In addition, we report the association between surgical treatment failure and biochemical markers of obesityrelated comorbidity. We also developed predictive models to identify patients with a high risk of surgical treatment failure 5 years postsurgery.

Results In total, $23.1 \%$ met at least one definition of surgical treatment failure at year 5 which was associated with (adjusted OR) with 95\% Cl): type 2 diabetes (T2D, OR $2.1 ; 95 \% \mathrm{Cl} 1.6$ to 2.7 ), dyslipidaemia (OR $1.8 ; 95 \% \mathrm{Cl}$ 1.6 to 2.1 ) and hypertension (OR $1.9 ; 95 \% \mathrm{Cl} 1.6$ to 2.2). Surgical treatment failure at 5 years was predicted by combined demographic and anthropometric measures from baseline, 1 and 2 years postsurgery (area under the curve $=0.874$ )

Conclusion Laparoscopic RYGB leads to a marked and sustained weight loss with improvement of obesity-related comorbidity in most patients. However, $23 \%$ met at least one definition of surgical treatment failure, which was associated with a greater risk of relapse and a higher incidence of T2D, dyslipidaemia and hypertension 5 years
Strengths and limitations of this study

- A large prospective cohort of nearly 6000 patients from bariatric surgery centres with a minimum of $60 \%$ retention rate at year 5 after bariatric surgery.

- Predefined thresholds of surgical treatment failure and cardiometabolic health were applied.

- The prediction model of surgical treatment failure was cross-validated using partial data, however, further validation of an unrelated cohort is preferable.

- Data originate from the whole of Sweden; thus generalisability may be limited to countries with similar ethnic diversity.

after surgery. Poor initial weight loss and early weight regain are strong predictors of long-term treatment failure and may be used for early identification of patients who require additional weight loss support.

\section{INTRODUCTION}

Obesity is a heterogeneous disease ${ }^{1}$ associated with several comorbid conditions, which ultimately increases the risk of all-cause mortality. ${ }^{2}$ Bariatric surgery is the most effective treatment for severe obesity. Long-term follow-up studies of Roux-en-Y gastric bypass (RYGB) show excellent results at the group level in reductions in weight, morbidity and mortality compared with non-surgical treatment. ${ }^{3-5}$ In Sweden, approximately 5500 bariatric operations are performed annually and, until 2014, the technique was almost exclusively RYGB. ${ }^{6}$

Weight loss after surgery is typically achieved during the first and second year, followed by weight maintenance or moderate regain 5-10 years after surgery. ${ }^{7}$ However, despite good overall results, the response and durability of surgically induced weight loss are heterogeneous ${ }^{8-10}$ and surgical treatment failure has been recognised as a potential clinical problem. ${ }^{11-13}$ 
The prevalence of surgical treatment failure is unclear, largely because an all-encompassing, unambiguous definition remains elusive. ${ }^{11-14}$ In a landmark controlled study by Adams et al based on 418 RYGB patients, $30 \%$ of participants experienced $<20 \%$ of total body weight loss at 12 years after RYGB.

It is still unclear to which extent cardiometabolic improvements after bariatric surgery depends on the degree of weight loss. Long-term studies have reported temporally declining rates of remission from obesityrelated comorbidities ${ }^{5}$ and the rate of relapse, especially for type 2 diabetes (T2D), has rather been attributed to pre-surgery disease duration and progression than to insufficient weight loss. ${ }^{516}$ Although an association between T2D relapse and weight regain has been suggested in some studies, ${ }^{17-19}$ others have not found any association between the degree of long-term weight loss and cardiometabolic outcome. ${ }^{20-22}$ The annual summary of the Scandinavian Obesity Surgery Registry (SOReg) recently described an association between baseline T2D and inadequate postoperative weight loss. ${ }^{23}$

In this study, based on a large cohort of patients prospectively collected in SOReg, ${ }^{6}$ we report on the heterogeneity of weight loss outcome, focusing primarily on the occurrence of surgical treatment failure 5years after surgery, according to any of three published definitions. We also report the association between surgical treatment failure and cardiometabolic disease and we present predictions of surgical treatment failure based on background data and weight development during the first 2 years after RYGB.

\section{METHODS}

\section{Data source}

The data source for this study was SOReg, a Swedish nationwide registry that began collecting data in 2007; from 2011, the registry covered 95\%-99\% of all bariatric surgery performed in Sweden. Between 2007 and 2011, RYGB constituted $96 \%-97 \%$ of all bariatric surgery performed. Data were retrieved in accordance with the study protocol. For this retrospective analysis, data were requested for all patients from surgical units and yearly cohorts that had a 5-year retention rate of $\geq 60 \%$. Data covered demographics, anthropometrics, pharmacological treatment, obesity-related comorbidity, biochemical markers and blood pressure at four time points: before surgery (baseline), and at 1, 2 and 5 years after surgery.

\section{Participants}

In total, 29 surgical units contributed data to the study through the SOReg database, ranging in number from 1 to 1643 patients, and data on 10633 unique patients were extracted.

Exclusion was performed in iteration steps and a total of 4697 patients were excluded.

The participants included in this study underwent RYGB during 2007-2011, 84.3\% had body mass index (BMI) reported for all time points. Missing data on BMI totalled $13.2 \%$ at either the 1-year or 2-year follow-up,

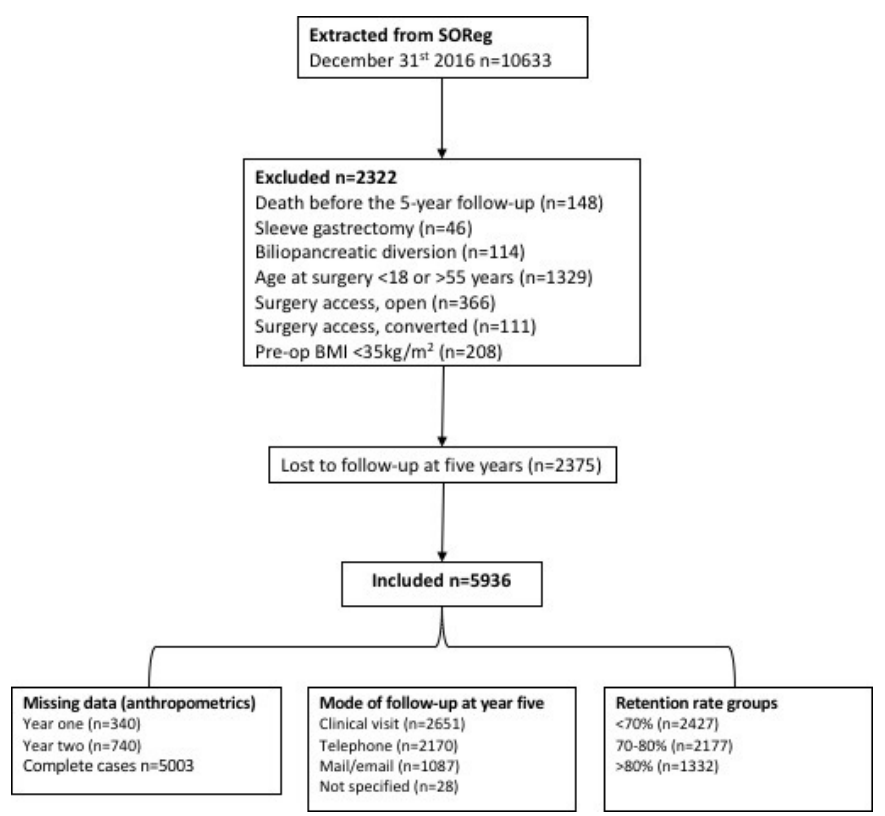

Figure 1 Flow chart of the study participants. BMI, body mass index; SOReg, Scandinavian Obesity Surgery Registry.

and $2.5 \%$ at both the 1-year and 2-year follow-ups. The follow-up modality at the 5-year follow-up was a clinical visit $(44.7 \%)$, telephone consultation $(36.6 \%)$, email/ letter $(18.3 \%)$ or unspecified $(0.5 \%)$. The follow-up modality at the 1-year and 2-year follow-ups are presented in detail in online supplemental eTable 1. Figure 1 shows the flow chart of the study participants.

\section{Loss to follow-up analysis}

A comparison of baseline characteristics between the study participants and those lost to follow-up revealed that lost participants had a younger age, a higher BMI and a male predomination. A detailed comparison appears in online supplemental eTable 2 in the Supplement.

\section{DEFINITIONS}

\section{Surgical treatment failure}

Surgical treatment failure was assessed and defined as meeting at least one of three definitions 5 years after surgery: $(1)<50 \%$ excess BMI loss (\%EBMIL), (2) $<20 \%$ total weight loss (\% TWL) and (3) BMI $>35 \mathrm{~kg} / \mathrm{m}^{2}$ where baseline was $<50 \mathrm{~kg} / \mathrm{m}^{2}$, or $>40 \mathrm{~kg} / \mathrm{m}^{2}$ if baseline BMI was $>50 \mathrm{~kg} / \mathrm{m}^{2}$. These definitions have been used elsewhere $^{1124}$ and, taken together, provide a means to define failure for patients within different weight categories.

\%EBMIL was calculated as ((baseline BMI - year 5 $\mathrm{BMI}) /($ baseline BMI - 25) $) * 100$.

\% TWL was calculated as ((baseline BMI - year 5 BMI) / baseline BMI)*100.

Two trajectories-inadequate weight loss and weight regain-can be defined that lead to long-term surgical treatment failure. Inadequate weight loss has been quantified during the first 6-12 months after surgery, ${ }^{25}$ and weight regain has typically been described as an increase above a specified threshold. ${ }^{12} 13$ 
In this paper, inadequate weight loss was defined as $<25 \%$ TWL from baseline to 1-year postsurgery, similar to the 25th percentile presented by Manning et $_{\text {al. }}{ }^{25}$

Early weight regain was defined as any absolute weight gain, expressed in kilograms, occurring between year 1 and 2 after surgery. This definition generated two groups. Long-term weight regain, defined according to Odom et $a l^{26}$ in three groups: $>15 \%$ regain of BMI nadir, $0.1 \%-$ $15 \%$ regain of BMI nadir and no weight regain, to 5 years postsurgery. These definitions were used to capture early weight regain as a predictive measurement of long-term surgical treatment failure, and to differentiate between the normally occurring fluctuation of body weight in the maintenance phase and the potentially harmful weight regain previously suggested. ${ }^{1819}$

For calculations, BMI nadir was accepted as the lowest measured weight at either the 1 or 2-year follow-up. In the case of missing data from one of those time points, the observed measurement was taken as the nadir.

\section{Obesity-related comorbidities and metabolic markers}

It is mandatory to report obesity-related comorbidities (eg, T2D, dyslipidaemia, hypertension $)^{6}$ requiring pharmacological treatment in SOReg, and data were available for $88 \%-100 \%$ included individuals depending on time point (full description in online supplemental eTable 3).

Blood pressure and biochemical markers, such as low-density lipoprotein, high-density lipoprotein, triglycerides, fasting glucose and glycated haemoglobin, are optional to report. Data were available data from $34 \%-73 \%$ included participants (online supplemental eTable 3).

Changes in blood pressure and biochemical markers were compared, stratified by surgical treatment failure at the 5-year follow-up, and by pharmacological treatment at baseline. Additionally, a broader classification of disease traits was generated, similar to that previously described, ${ }^{527}$ by compiling a disease-specific biochemical marker above a cut-off (online supplemental eAppendix 1), in combination with pharmacological treatment. This classification was applied at all time points and used to assess prevalence and change over time. Thus, six groups were generated: participants without disease traits at baseline were classified 'disease-free' if no disease trait was evident at any time point, 'intermittent' if disease-free at both baseline and 5-year follow-up, but not in between, and 'incidence' where a disease trait developed during the 5-year follow-up period. Participants with a disease trait at baseline were classified 'remission' if no disease trait was evident at 5-year follow-up, 'relapse' if disease-free at year 1, 2 or both, but not at year 5 , and 'no remission' where at least one disease trait was evident at all time points.

For clarity, the compiled disease traits are hereafter referred to as T2D, dyslipidaemia and hypertension.

\section{Statistics}

All statistical analyses were performed using SPSS V.24 (IBM) and STATA IC V.15.1 (Stata). Descriptive statistics are presented as mean \pm standard deviation $( \pm \mathrm{SD})$, or as a percentage $(\%)$, unless otherwise specified.

Characteristics were compared between those lost to follow-up (online supplemental eTable 2) and those included in the analysis, as well as according to surgical treatment failure status (online supplemental eTable 4), using independent t-test and $\chi^{2}$ test.

We described the prevalence and change in cardiometabolic disease and assessed the odds associated with surgical treatment failure using logistic regression, first using a crude model (data not shown) and then multivariable models (separate, compiled or additive for each definition of surgical treatment failure) in which we adjusted for sex, age and BMI at baseline and corresponding cardiometabolic disease. Results are presented as OR with $95 \%$ CIs.

In addition, we used logistic regression to predict the probability of meeting at least one definition of surgical treatment failure, which we considered dichotomously ( 1 =surgical treatment failure, $0=$ otherwise). Our predictions used sex, baseline, age, BMI and \% TWL for the first year and change in weight $(\mathrm{kg})$ for the second year. We measured performance by calculating the receiver operating characteristic curve and the corresponding area under the curve (AUC) and by using cross-validation (leave $10 \%, \mathrm{k}=10$ replicates).

Finally, several sensitivity analyses were undertaken for the primary endpoint (ie, surgical treatment failure), which can be found in online supplemental eAppendix 2.

The significance level was set to 0.05 for all analyses (two tailed), and $\mathrm{p}$ values are reported with three decimals.

\section{Patient and public involvement}

Patients nor the public were involved in the conduct of this study.

\section{RESULTS}

In total, 5936 patients ( $79.1 \%$ female), aged $18-55$ years, who had undergone laparoscopic RYGB (LRYGB) from 2007 to 2011, were included in the final sample (figure 1). At baseline, the mean age was $39.4 \pm 9.0$ years and BMI was $42.9 \pm 5.1 \mathrm{~kg} / \mathrm{m}^{2}$. Patient characteristics are presented in table 1. At year 5, overall mean BMI was $30.4 \pm 5.2$, mean weight loss $35.8 \pm 13.8 \mathrm{~kg}$, BMIL $12.6 \pm 4.7 \mathrm{~kg} / \mathrm{m} 2$, \% EBMIL $72.2 \pm 25.2 \%$ and $\%$ TWL $29.1 \pm 9.8 \%$.

Inadequate weight loss (ie, $<25 \%$ TWL from baseline to year 1) was identified in $17.1 \%$ of 5596 participants with available data.

Early weight regain (between year 1 and 2) was identified in $38.7 \%$ of 5010 participants with available data, with a mean increase of $4.5 \pm 3.9 \mathrm{~kg}$ (range 1-38 kg), compared with a mean decrease of $4.4 \pm 5.1 \mathrm{~kg}$ (range $66-0 \mathrm{~kg}$ ) in the no regain group.

Long-term weight change between nadir and 5-year follow-up was distributed as follows: $>15 \%$ regain $(+17.7 \pm 7.2 \mathrm{~kg}$, range $7-101 \mathrm{~kg})$ in $19.9 \%$ of participants, $0.1 \%-15 \%$ regain $(+5.7 \pm 3.5 \mathrm{~kg}$, range 0 to $19 \mathrm{~kg})$ in $59.3 \%$ 


\begin{tabular}{|c|c|c|}
\hline & $\mathbf{n}$ & Mean (SD) \\
\hline Age at surgery & 5936 & $39.4(9.0)$ \\
\hline Sex, no \% female & 5936 & 79.1 \\
\hline Height, cm & 5936 & $168.8(8.9)$ \\
\hline Weight, kg & 5936 & $122.8(20.0)$ \\
\hline Body mass index at surgery, $\mathrm{kg} / \mathrm{m}^{*}$ & 5936 & $42.9(5.1)$ \\
\hline \multicolumn{3}{|l|}{ Glucose metabolism } \\
\hline Glucose, mmol/L & 2861 & $5.9(1.9)$ \\
\hline $\mathrm{HbA} 1 \mathrm{c}, \mathrm{mmol} / \mathrm{mol}$ & 4168 & $40.6(11.4)$ \\
\hline $\begin{array}{l}\text { Pharmacological diabetes } \\
\text { treatment, no (\%) }\end{array}$ & 5936 & $675(11.4)$ \\
\hline Diabetes type $2 \dagger$, no (\%) & 5936 & 89615.1 \\
\hline \multicolumn{3}{|l|}{ Lipids } \\
\hline $\begin{array}{l}\text { High-density lipoprotein (HDL), } \\
\mathrm{mmol} / \mathrm{L}\end{array}$ & 4188 & $1.2(0.4)$ \\
\hline $\begin{array}{l}\text { Low-density lipoprotein (LDL), } \\
\mathrm{mmol} / \mathrm{L}\end{array}$ & 4110 & $3.1(0.9)$ \\
\hline Triglycerides (TG), mmol/L & 4314 & $1.7(1.4)$ \\
\hline $\begin{array}{l}\text { Pharmacological dyslipidaemia } \\
\text { treatment, no (\%) }\end{array}$ & 5936 & $414(7.0)$ \\
\hline Dyslipidaemiał, no (\%) & 5936 & $3601(67.5)$ \\
\hline \multicolumn{3}{|l|}{ Physiology } \\
\hline Systolic BP, mm Hg & 2960 & $133(16)$ \\
\hline Diastolic BP, mm Hg & 2960 & $83(10)$ \\
\hline $\begin{array}{l}\text { Pharmacological hypertension } \\
\text { treatment, no (\%) }\end{array}$ & 5936 & $1158(19.5)$ \\
\hline Hypertension $\ddagger$ no. (\%) & 5936 & $1683(28.4)$ \\
\hline
\end{tabular}

*Pharmacologically treated T2D/fasting glucose $>7.0 \mathrm{mmol} / \mathrm{L}$ $\mathrm{HbA} 1 \mathrm{c}>48 \mathrm{mmol} / \mathrm{mol}$.

†Pharmacologically treated dyslipidaemia/LDL $>4.1 / \mathrm{TG}>2.0 / \mathrm{HDL}$ $<1 \mathrm{mmol} / \mathrm{L}$ for males and $<1.3 \mathrm{mmol} / \mathrm{L}$ for females.

$\ddagger$ Pharmacologically treated blood pressure/systolic $\geq 140 \mathrm{~mm} \mathrm{Hg}$ or diastolic blood pressure $>90 \mathrm{~mm} \mathrm{Hg}$.

BP, blood pressure; HbA1c, glycated haemoglobin; T2D, type 2 diabetes.

and no weight regain $(-5.0 \pm 5.2 \mathrm{~kg}$, range -36 to $0 \mathrm{~kg})$ in $20.8 \%$.

Overall, the prevalence of meeting at least one of the three definitions of surgical treatment failure 5 years after LRYGB was $23.1 \% \quad(\mathrm{n}=1371)$. The distribution between the three definitions was $19.2 \%(\mathrm{n}=1138)$ for $<50 \%$ EBMIL, $17.0 \%(\mathrm{n}=1010)$ for $<20 \%$ TWL and $14.1 \%$ $(\mathrm{n}=835)$ for $\mathrm{BMI}>35$ or $>40 \mathrm{~kg} / \mathrm{m}^{2}$. There was substantial overlap, $39.8 \% \quad(\mathrm{n}=545)$ meeting all three definitions and $38.1 \%(\mathrm{n}=522)$ meeting two of the three definitions (figure 2).

Surgical treatment failure was more common among patients with inadequate weight loss $(60 \%$ vs $15.4 \%$, $\mathrm{p}<.001)$ and early weight regain $(33.8 \%$ vs $15.6 \%, \mathrm{p}<.001)$. Comparing long-term weight regain, the proportion meeting criteria for failure was highest in participants

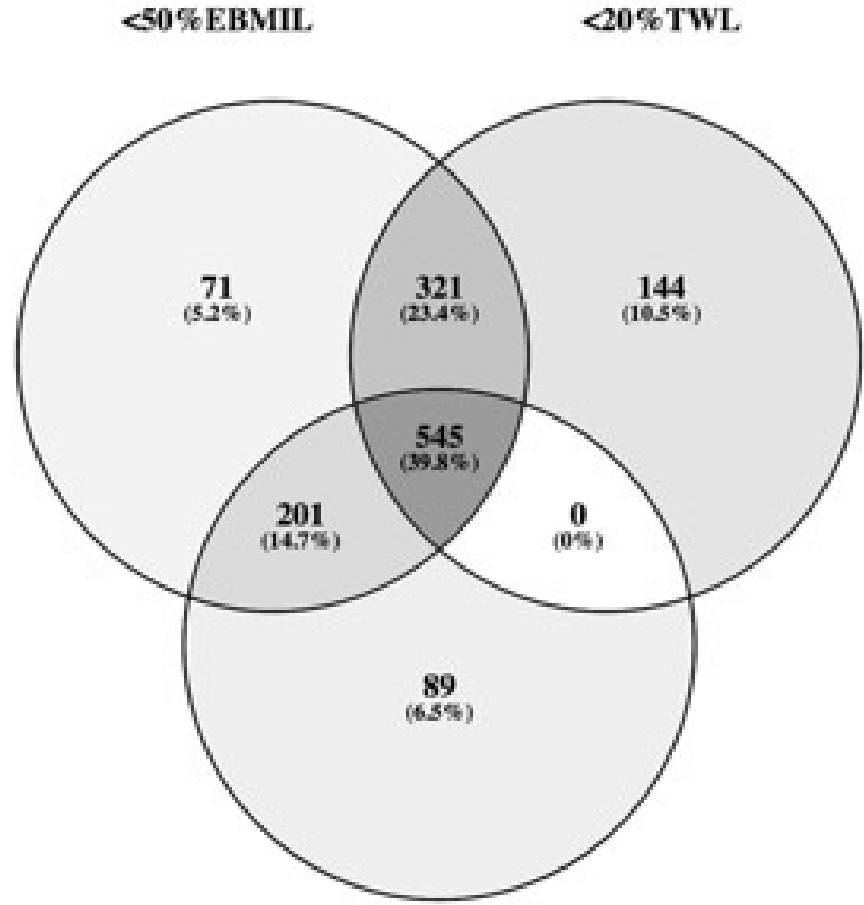

with $>15 \%$ regain from nadir $(46.7 \%)$, followed by $0.1 \%-$ $15 \%(21.1 \%)$ and no regain $(5.1 \%),(\mathrm{p}<.001)$. Patients with no long-term weight regain but surgical treatment failure $(\mathrm{n}=59)$ had higher baseline BMI (48.5 vs 43.1, $\mathrm{p}<.001)$ and lower \% TWL at 1-year and 2-year follow-up $(-18.0 \%$ vs $-30.5 \%$ and $-18.1 \%$ vs $-32.3 \%$, respectively, both $\mathrm{p}<.001)$.

\section{Cardiometabolic disease}

Biochemical and physiological measures improved following surgery in participants with and without surgical treatment failure. Mean values, stratified by surgical treatment failure and baseline pharmacological treatment, are shown from baseline to year 5 in online supplemental eFigures $1 \mathrm{~A}-\mathrm{G}$ and $2 \mathrm{~A}-\mathrm{G}$.

Overall, the prevalence of cardiometabolic disease decreased from baseline to 5 years: T2D from $15.1 \%$ $(\mathrm{n}=896)$ to $6.4 \% \quad(\mathrm{n}=380)$, dyslipidaemia from $60.7 \%$ $(\mathrm{n}=3603)$ to $16.4 \% \quad(\mathrm{n}=974)$, and hypertension from $28.4 \%(\mathrm{n}=1683)$ to $18.9 \%(\mathrm{n}=1124)$. The rates of being disease-free, incident and intermittent disease, as well as remission, relapse and no remission, varied between surgical and non-surgical treatment failure (table 2).

Logistic regression (adjusted for sex, age, BMI and corresponding cardiometabolic disease at baseline) confirmed an association between surgical treatment failure and cardiometabolic disease at year 5: T2D, 
Table 2 Change in cardiometabolic disease status from baseline to 5 years postsurgery compared between surgical treatment failure (STF) and non-STF

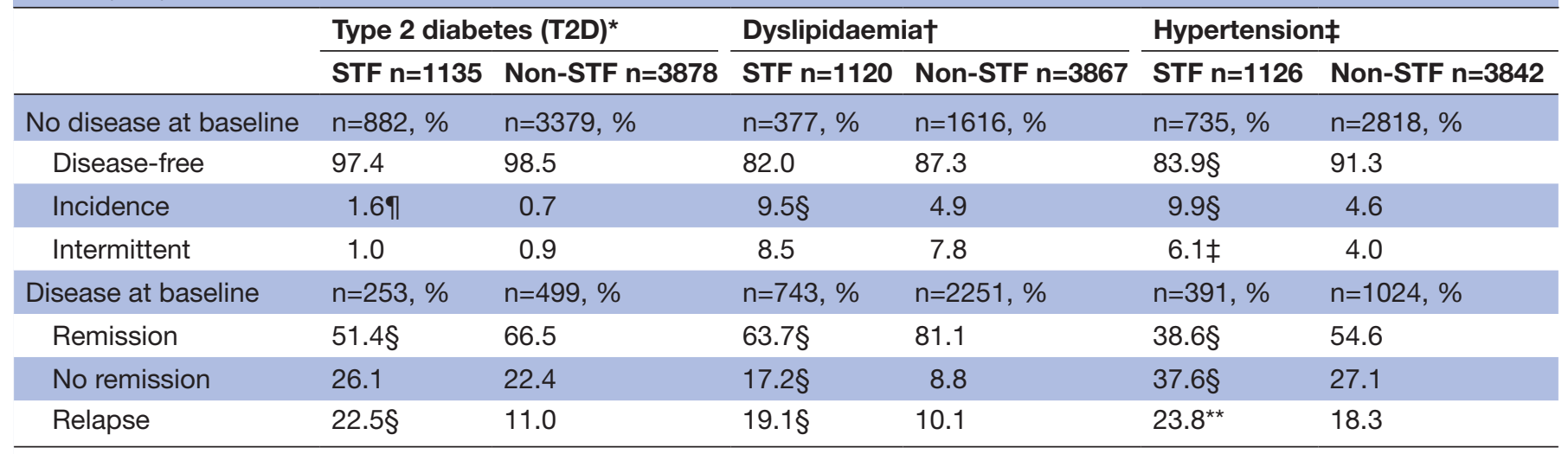

${ }^{*}$ Pharmacologically treated T2D/fasting glucose $>7.0 \mathrm{mmol} / \mathrm{L} / \mathrm{HbA} 1 \mathrm{c}>48 \mathrm{mmol} / \mathrm{mol}$.

†Pharmacologically treated dyslipidaemia/LDL $>4.1 / \mathrm{TG}>2.0 / \mathrm{HDL}<1 \mathrm{mmol} / \mathrm{L}$ for males and $<1.3 \mathrm{mmol} / \mathrm{L}$ for females.

$\ddagger$ Pharmacologically treated blood pressure/systolic $\geq 140 \mathrm{~mm} \mathrm{Hg}$ or diastolic blood pressure $>90 \mathrm{~mm} \mathrm{Hg}$.

§Indicates a statistically significant difference at $p<.001$.

IIIndicates a statistically significant difference at $p<.010$.

${ }^{* *}$ Indicates a statistically significant difference at $p<.001$.

HDL, high-density lipoprotein; LDL, low-density lipoprotein; T2D, type 2 diabetes; TG, triglycerides.

OR 2.10 (95\% CI 1.61 to 2.75); dyslipidaemia, OR 2.50 (95\% CI 2.14 to 2.92) and hypertension, OR 1.85 (95\% CI 1.55 to 2.21). Individual definitions were similarly associated with cardiometabolic disease (online supplemental eTable 5). The combined effect of fulfilling one, two or three of the definitions is presented in online supplemental eTable 6. Predicted probability of cardiometabolic disease plotted against continuous \%EBMIL, \% TWL and BMI at year 5 is illustrated in online supplemental eFigures 3-5A-C.

Inadequate weight loss during year 1 was significantly associated with T2D (OR 1.84; 95\% CI 1.38 to 2.45), dyslipidaemia (OR 1.89; 95\% CI 1.59 to 2.25 ) and hypertension (OR 1.61; 95\% CI 1.32 to 1.96$)$. Late weight regain ( $\geq 15 \%$ regain from nadir) was significantly associated with dyslipidaemia (OR 1.64; 95\% CI 1.31 to 2.05) and hypertension (OR $1.41 ; 95 \%$ CI 1.10 to 1.81 ), but not T2D (OR 1.25; $95 \%$ CI 0.84 to 1.88 ).

\section{Predicting surgical treatment failure}

The estimated regression coefficients and OR are presented in table 3. Given age, sex and baseline BMI and a negatively expressed value of $\% \mathrm{TWL}$ from baseline to the 1-year follow-up, and change in weight $(\mathrm{kg})$ between 1-year and 2-year follow-up, the predicted probability of surgical treatment failure 5 years after surgery is given by:

$\mathrm{P}($ surgical treatment failure $)=\exp (\mathrm{a}) /(1+(\mathrm{a}))$ with $\mathrm{a}=$ $-1.1+-0.00545 *($ sex male $=0$ female $=1)+0.00299 *$ (age at surgery,years $)+0.14949 *($ baseline BMI $)+0.22310 *$ (\% TWL year 1$)+0.15982 *$ (weight change year 1 to year $2(\mathrm{~kg})$ ). Examples of the probability calculation are presented in online supplemental eAppendix 3.

As depicted in figure 3 , this simple model provided a good prediction $(\mathrm{AUC}=0.8743)$.

\section{DISCUSSION}

This analysis of prospectively collected data on 5963 adults who underwent primary LRYGB surgery, revealed that almost one in four participants fulfilled at least one of the three applied definitions of surgical treatment failure, 5 years after surgery. Surgical treatment failure was associated with a negative effect on cardiometabolic health: lower rate of remission and more frequent relapse and incidence of T2D, dyslipidaemia and hypertension.

Table 3 Final multivariable model for predicting surgical treatment failure 5 years after surgery

\begin{tabular}{lrrrrrr}
\hline & Beta (B) & SE & Wald & P value & Exp(B) & 95\% Cl \\
\hline Sex $(0=$ male) & -0.00545 & 0.099 & 0.003 & 0.956 & 0.995 & 0.818 to 1.209 \\
Age at surgery, years & 0.00299 & 0.005 & 0.361 & 0.548 & 1.003 & 0.993 to 1.013 \\
BMI at surgery, $\mathrm{kg} / \mathrm{m}^{2}$ & 0.14949 & 0.009 & 283.640 & 0.000 & 1.161 & 1.141 to 1.182 \\
Percentage BMI loss during year 1, \%TWL & 0.22310 & 0.008 & 794.848 & 0.000 & 1.250 & 1.231 to 1.269 \\
Change in weight between year 1 and 2, kg & 0.15982 & 0.008 & 382.606 & 0.000 & 1.173 & 1.155 to 1.192 \\
Intercept & -1.09588 & 0.513 & 4.569 & 0.033 & 0.334 & \\
\hline
\end{tabular}

BMI, body mass index; TWL, total weight loss. 


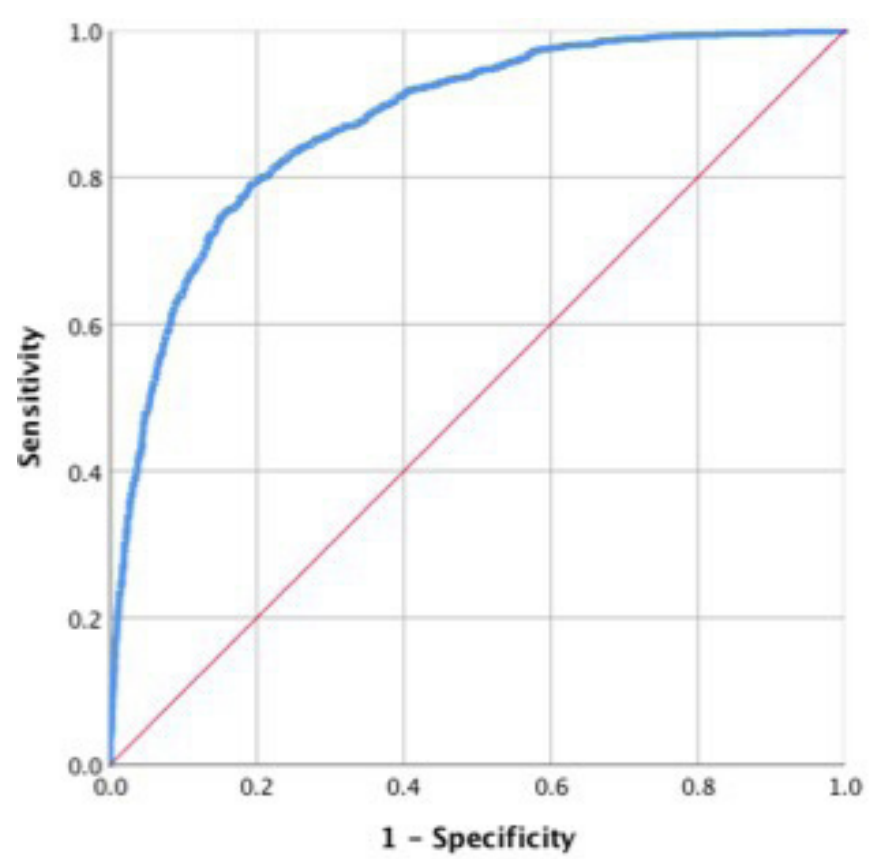

Figure 3 Receiver operating characteristic curve with predicted probability of surgical treatment failure, given age, sex and baseline BMI and \%TWL from baseline to the 1-year follow-up and change in weight $(\mathrm{kg})$ between year 1 and year 2 follow-ups: area under the curve $=0.8743$ (95\% Cl 0.8630 0.8856). BMI, body mass index; TWL, total weight loss.

Each definition of surgical treatment failure and weight regain was independently associated with cardiometabolic health. In total, $9.2 \%$ of the study population fulfilled the criteria for all of the three definitions ${ }^{1124}$ of surgical treatment failure and they provided a very strong association with T2D, dyslipidaemia and hypertension.

The extent to which insufficient weight loss and weight regain affect cardiometabolic outcome is unclear, both confirmative $^{13} 181928-30$ and negative ${ }^{20-22} 3132$ findings have been reported. In the present study inadequate weight loss during year 1 and weight regain during year 2 were investigated. Both were found to be associated with cardiometabolic outcomes, however, both were in the present study viewed as prerequisites for surgical treatment failure, which in turn was associated with a less favourable metabolic profile 5 years after surgery, regardless of whether or not patients were taking T2D, dyslipidaemia or hypertension medications prior to surgery.

Early identification of those with a high risk of long-term surgical treatment failure may facilitate additional weight loss support. ${ }^{33-35}$ Unfortunately neither we, nor others, have been able to build a sufficiently reliable model using exclusively presurgical characteristics. ${ }^{36}$ However, our results indicate that long-term surgical treatment failure can, with good accuracy ( $\mathrm{AUC}=0.8743$ ), be predicted by sex, age and BMI at baseline, together with \% TWL during year 1 and weight change during year 2 . We found that \% TWL during year 1 was the strongest predictor of surgical treatment failure. Similarly the initial 6-month weight loss predicts the 24 -month weight loss. ${ }^{25}$ Of note, we found that presurgical T2D, dyslipidaemia and hypertension were associated with surgical treatment failure, a finding that may warrant further research as the associations could be dependent on both behavioural and physiological factors.

The present study terms long-term poor weight loss after surgery as surgical treatment failure. This wording should not be interpreted to mean that the surgical procedure failed, but rather that the therapy alone was insufficient to produce the required degree of long-term weight loss. This reasoning should not be surprising given the heterogeneous nature of obesity, as any standardised treatment is likely to result in a spectrum of outcomes. Despite that, bariatric surgery has remained a stand-alone treatment. This is contrary to bariatric surgery guidelines suggesting active treatment of patients with poor weight outcome. ${ }^{37}$ In addition patients have also expressed a need for more extensive follow-up. ${ }^{38}$ Recognising this, bariatric surgery would likely benefit from the application of the multidisciplinary and multimodal approach that has evolved in other fields of disease, such as cancer care, where for decades surgery has been integrated into multimodal treatment pathways, alongside chemotherapies and radiation therapies. It has been shown that behavioural support ${ }^{35}$ and pharmacological treatment $^{34}$ can improve the outcome after surgery, indicating potential for additive, perhaps even synergistic effects of combination therapies. However, as a consequence of the disintegrated follow-up after surgery, it is still unclear to which extent outcome after bariatric surgery can be optimised by means of adjuvant treatment.

Strengths of this study include SOReg's prospective collection of data from the whole of Sweden, with broad national coverage. This was demonstrated by the inclusion of nearly 6000 patients from the database of centres with $\mathrm{a} \geq 60 \%$ retention rate 5 years after LRYGB, providing a large and robust data set permitting subgroup analysis. All patients included in the final sample had undergone LRYGB. This constituted $95 \%-97.5 \%$ of all bariatric surgery performed between 2007 and 2011 in Sweden, thus reducing possible bias in patient selection for different surgical procedures.

There are also some limitations. Although the impact of surgical treatment failure on metabolic health is substantial, it does not account for all comorbidity seen at the 5-year follow-up. Other factors, such as disease duration before surgery, are also of importance but such information was not available in this study. Neither was information on psychological disorders available, thus limiting the possibility to evaluate and include such factors in the prediction model.

Missing data analysis revealed that rates of surgical treatment failure at year 1 and 2 were higher in the $28.6 \%$ that were lost to follow-up year 5 , indicating that the actual proportion of surgical treatment failure may be higher than what the results suggests. In addition, there was a difference in weight loss between the modes of follow-up, possibly implying bias of self-reported data. Similarly, a 
statistical limitation of note is that we compiled diseasespecific traits where missing data are implicitly treated as zeroes. For example, the estimated effects may be diluted (biased towards zero) because the comparison is actual ones vs a mixture of zeroes and ones. Thus, both the overall prevalence of surgical treatment failure and cardiometabolic disease may be underestimated.

The developed prediction model for long-term surgical treatment failure was cross-validated using partial data and can readily be applied to countries with similar cultural and ethnic settings as in northern Europe. However, further validation of an unrelated cohort is preferable, and further devolvement of the model may be required to encompass ethnic diversity.

Unsuccessful surgical treatment result is difficult to define and a large number of definitions and time points have been used. ${ }^{11-14}$ Our results would probably have been slightly modified if we had used other definitions. However, the strong associations between surgical treatment failure, as defined in the present study, and cardiometabolic health may support their clinical usefulness.

\section{CONCLUSION}

RYGB is associated with improvement of obesity-related comorbidity. However, 23\% of the patients developed surgical treatment failure 5years after surgery, which was associated with a markedly increased risk of cardiometabolic disease. Initial weight loss and early weight regain were strong predictive markers that can be used for the early identification of patients with a high risk of long-term failure. This study underlines the need for long-term follow-up of patients undergoing bariatric surgery by a multidisciplinary team and improved additional behavioural and pharmacological treatment postsurgery are warranted.

\section{Author affiliations \\ ${ }^{1}$ Department of Clinical Science, Intervention and Technology, Division of Pediatrics, KI CLINTEC, Huddinge, Sweden \\ ${ }^{2}$ Allied Health Professionals Function, Occupational Therapy \& Physiotherapy, Karolinska University Hospital, Stockholm, Sweden \\ ${ }^{3}$ Department of Surgical Research, University of Gothenburg Institute of Clinical Sciences, Gothenburg, Sweden \\ ${ }^{4}$ Swansea University Medical School, Swansea, UK \\ ${ }^{5}$ Department of Biomedical and Clinical Sciences, Linkoping University, Linkoping, Sweden \\ ${ }^{6}$ Department of Surgery, Vrinnevi Hospital in Norrkoping, Norrkoping, Sweden}

Contributors MB and CM conceptualised the study, MB performed data and statistical analyses and drafted the manuscript. All authors (MB, AJB, T0 and CM) contributed to result interpretation and critically revised and approved the final version of the manuscript. MB and CM had full access to all the data in the study and takes responsibility for the integrity of the data and the accuracy of the data analysis.

Funding This work was funded by the Swedish order of Freemasons (grant no: not applicable), the Swedish Heart- Lung Foundation (grant no: 20180581), the Samariten foundation for pediatric research (grant no: not applicable) and AnnaLisa and Arne Gustafssons foundation (grant no: not applicable).

Disclaimer The funders of this study had no part in study design, collection, analysis or interpretation of data, nor in the writing of the report or in the decision to submit the paper for publication. The corresponding author had full access to the data and had final responsibility for the decision to submit for publication.
Competing interests TO declares participation in advisory board for J\&J and Novo Nordisk and reimbursement for lectures and education activities. All fees to institution. CM has received research grants from Novo Nordisk, Sigrid THX AB and salaries as medical advisor for Itrim $A B$ and Weight Watchers Int. MB and AJB declares no conflict of interest.

Patient consent for publication Not required.

Ethics approval This study was approved by the Stockholm ethical board (2017/1793-31).

Provenance and peer review Not commissioned; externally peer reviewed.

Data availability statement Data may be obtained from a third party and are not publicly available. All data relevant to the study are included in the article or uploaded as online supplemental information. No additional data available from the authors. Original data may be requested from the Scandinavian Obesity Surgery Registry (https://www.ucr.uu.se/soreg/in-english)

Supplemental material This content has been supplied by the author(s). It has not been vetted by BMJ Publishing Group Limited (BMJ) and may not have been peer-reviewed. Any opinions or recommendations discussed are solely those of the author(s) and are not endorsed by BMJ. BMJ disclaims all liability and responsibility arising from any reliance placed on the content. Where the content includes any translated material, BMJ does not warrant the accuracy and reliability of the translations (including but not limited to local regulations, clinical guidelines, terminology, drug names and drug dosages), and is not responsible for any error and/or omissions arising from translation and adaptation or otherwise.

Open access This is an open access article distributed in accordance with the Creative Commons Attribution 4.0 Unported (CC BY 4.0) license, which permits others to copy, redistribute, remix, transform and build upon this work for any purpose, provided the original work is properly cited, a link to the licence is given, and indication of whether changes were made. See: https://creativecommons.org/ licenses/by/4.0/.

ORCID iD

Markus Brissman http://orcid.org/0000-0003-1971-6431

\section{REFERENCES}

1 González-Muniesa P, Mártinez-González M-A, Hu FB, et al. Obesity. Nat Rev Dis Primers 2017;3:17034.

2 Aune D, Sen A, Prasad M, et al. BMI and all cause mortality: systematic review and non-linear dose-response meta-analysis of 230 cohort studies with 3.74 million deaths among 30.3 million participants. BMJ 2016;353:i2156.

3 Sjöström L. Review of the key results from the Swedish Obese Subjects (SOS) trial - a prospective controlled intervention study of bariatric surgery. J Intern Med 2013;273:219-34.

4 Gloy VL, Briel M, Bhatt DL, et al. Bariatric surgery versus nonsurgical treatment for obesity: a systematic review and meta-analysis of randomised controlled trials. BMJ 2013;347:f5934.

5 Adams TD, Davidson LE, Litwin SE, et al. Weight and metabolic outcomes 12 years after gastric bypass. N Engl J Med 2017;377:1143-55.

6 Hedenbro JL, Näslund E, Boman L, et al. Formation of the Scandinavian obesity surgery registry, SOReg. Obes Surg 2015;25:1893-900.

7 Buchwald H, Avidor Y, Braunwald E, et al. Bariatric surgery: a systematic review and meta-analysis. JAMA 2004;292:1724-37.

8 de Hollanda A, Ruiz T, Jiménez A, et al. Patterns of weight loss response following gastric bypass and sleeve gastrectomy. Obes Surg 2015;25:1177-83.

9 Azagury D, Mokhtari TE, Garcia L, et al. Heterogeneity of weight loss after gastric bypass, sleeve gastrectomy, and adjustable gastric banding. Surgery 2019;165:565-70.

10 Courcoulas AP, King WC, Belle SH, et al. Seven-Year weight trajectories and health outcomes in the longitudinal assessment of bariatric surgery (Labs) study. JAMA Surg 2018;153:427-34.

11 Mann JP, Jakes AD, Hayden JD, et al. Systematic review of definitions of failure in revisional bariatric surgery. Obes Surg 2015;25:571-4.

12 Karmali S, Brar B, Shi X, et al. Weight recidivism post-bariatric surgery: a systematic review. Obes Surg 2013;23:1922-33.

13 King WC, Hinerman AS, Belle SH, et al. Comparison of the performance of common measures of weight regain after bariatric surgery for association with clinical outcomes. JAMA 2018;320:1560-9. 
14 Bonouvrie DS, Uittenbogaart M, Luijten AAPM, et al. Lack of Standard Definitions of Primary and Secondary (Non)responders After Primary Gastric Bypass and Gastric Sleeve: a Systematic Review. Obes Surg 2019;29:691-7.

15 Sjöström L, Lindroos A-K, Peltonen M, et al. Lifestyle, diabetes, and cardiovascular risk factors 10 years after bariatric surgery. $N$ Engl J Med 2004;351:2683-93.

16 Pories WJ, Swanson MS, MacDonald KG, et al. Who would have thought it? An operation proves to be the most effective therapy for adult-onset diabetes mellitus. Ann Surg 1995;222:339-52.

17 Shah M, Simha V, Garg A. Review: long-term impact of bariatric surgery on body weight, comorbidities, and nutritional status. J Clin Endocrinol Metab 2006;91:4223-31.

18 DiGiorgi M, Rosen DJ, Choi JJ, et al. Re-emergence of diabetes after gastric bypass in patients with mid- to long-term follow-up. Surg Obes Relat Dis 2010;6:249-53.

19 Laurino Neto RM, Herbella FAM, Tauil RM, et al. Comorbidities remission after Roux-en-Y gastric bypass for morbid obesity is sustained in a long-term follow-up and correlates with weight regain. Obes Surg 2012;22:1580-5.

20 Hawkins RB, Mehaffey JH, McMurry TL, et al. Clinical significance of failure to lose weight 10 years after Roux-en-Y gastric bypass. Surg Obes Relat Dis 2017;13:1710-6.

21 Magno FCCM, Sousa PAMde, Rodrigues MP, et al. Long term maintenance of glucose and lipid concentrations after Roux-en- $Y$ gastric bypass. Arch Endocrinol Metab 2018;62:346-51.

22 Aminian A, Jamal M, Augustin T, et al. Failed surgical weight loss does not necessarily mean failed metabolic effects. Diabetes Technol Ther 2015; 17:682-4.

23 Registry TSOS. Fördjupningsrapport SOReg 2019, diabetes, 2019. Available: https://www.ucr.uu.se/soreg/arsrapporter [Accessed 28 oct 2019].

24 Corcelles R, Boules M, Froylich D, et al. Total weight loss as the outcome measure of choice after Roux-en-Y gastric bypass. Obes Surg 2016;26:1794-8

25 Manning S, Pucci A, Carter NC, et al. Early postoperative weight loss predicts maximal weight loss after sleeve gastrectomy and Rouxen-Y gastric bypass. Surg Endosc 2015;29:1484-91.

26 Odom J, Zalesin KC, Washington TL, et al. Behavioral predictors of weight regain after bariatric surgery. Obes Surg 2010;20:349-56.
27 Inge TH, Courcoulas AP, Jenkins TM, et al. Five-year outcomes of gastric bypass in adolescents as compared with adults. $N$ Engl J Med 2019;380:2136-45.

28 Zhou K, Wolski K, Malin SK, et al. Impact of weight loss trajectory following randomization to bariatric surgery on long-term diabetes glycemic and cardiometabolic parameters. Endocr Pract 2019;25:572-9.

29 Nicoletti CF, de Oliveira BAP, de Pinhel MAS, et al. Influence of excess weight loss and weight regain on biochemical indicators during a 4-year follow-up after Roux-en-Y gastric bypass. Obes Surg 2015;25:279-84.

30 Brethauer SA, Aminian A, Romero-Talamás H, et al. Can diabetes be surgically cured? long-term metabolic effects of bariatric surgery in obese patients with type 2 diabetes mellitus. Ann Surg 2013;258:628-37.

31 Cohen RV, Rubino F, Schiavon C, et al. Diabetes remission without weight loss after duodenal bypass surgery. Surg Obes Relat Dis 2012;8:e66-8.

32 Cohen RV, Pinheiro JC, Schiavon CA, et al. Effects of gastric bypass surgery in patients with type 2 diabetes and only mild obesity. Diabetes Care 2012;35:1420-8.

33 Kalarchian MA, Marcus MD. Psychosocial interventions pre and post bariatric surgery. Eur Eat Disord Rev 2015;23:457-62.

34 Nor Hanipah Z, Nasr EC, Bucak E, et al. Efficacy of adjuvant weight loss medication after bariatric surgery. Surg Obes Relat Dis 2018;14:93-8.

35 Andreu A, Jimenez A, Vidal J, et al. Bariatric support groups predicts long-term weight loss. Obes Surg 2020;30:2118-23.

36 Livhits M, Mercado C, Yermilov I, et al. Preoperative predictors of weight loss following bariatric surgery: systematic review. Obes Surg 2012;22:70-89.

37 Mechanick JI, Youdim A, Jones DB, et al. Clinical practice guidelines for the perioperative nutritional, metabolic, and nonsurgical support of the bariatric surgery patient-2013 update: cosponsored by American Association of Clinical Endocrinologists, The Obesity Society, and American Society for Metabolic \& Bariatric Surgery. Obesity 2013;21:S1-27.

38 Parretti HM, Hughes CA, Jones LL. 'The rollercoaster of follow-up care' after bariatric surgery: a rapid review and qualitative synthesis. Obes Rev 2019;20:88-107. 